\title{
COMPLICAÇÕES VASCULARES PÓS-TRANSPLANTE HEPÁTICO
}

\author{
VASCULAR COMPLICATIONS AFTER LIVER TRANSPLANTATION
}

\author{
Júlio Cezar Uili Coelho, TCBC-PR ${ }^{1}$ \\ Jorge Eduardo Fouto Matias ${ }^{2}$ \\ Mônica Beatriz Parolin² \\ Eduardo Lopes Martins, TCBC-PR ${ }^{2}$ \\ Paolo Rogério de Oliveira Salvalaggio, ACBC-PR $^{3}$ \\ Carolina Gomes Gonçalves ${ }^{4}$
}

\begin{abstract}
RESUMO: Objetivo: O objetivo do estudo é apresentar as complicações vasculares arteriais e venosas observadas em um serviço de transplante hepático universitário brasileiro. Métodos: Os prontuários de todos os pacientes submetidos a transplante hepático no período de setembro de 1991 a janeiro de 2000 foram analisados para determinar as complicações vasculares e correlacioná-las a dados clínicos e do procedimento cirúrgico. Resultados: Foram realizados 169 transplantes, sendo quatro inter vivos e nove retransplantes. Um total de 24 complicações vasculares $(14,3 \%)$ foi identificado em 22 pacientes (13,0\%). A complicação vascular mais comum foi a trombose da artéria hepática (15 casos), seguido da trombose da veia porta (quatro casos). Complicações da veia cava inferior infra ou supra-hepática foram incomuns, ocorrendo em um total de três casos $(1,8 \%)$. As complicações vasculares foram mais freqüentes nas crianças $(26,06 \%)$ do que em adultos $(13,14 \%)(\mathrm{p}<0,05)$. Dos pacientes com trombose da artéria hepática, um foi submetido à angioplastia, um a trombectomia, oito a retransplante e cinco evoluíram para o óbito enquanto aguardavam retransplante. Dos quatro casos de trombose da veia porta, dois foram a óbito, um foi submetido à colocação percutânea de prótese e um a tratamento conservador. Os pacientes com estenose da veia porta e da veia cava inferior infra e supra-hepática foram submetidos a tratamento conservador, com boa evolução clínica. Conclusões: que as complicações vasculares são freqüentes após o transplante hepático, principalmente em crianças, e são associadas à elevada morbidade, mortalidade e retransplante.
\end{abstract}

Descritores: Transplante hepático; Complicações vasculares; Trombose venosa; Trombose arterial.

\section{INTRODUÇÃO}

Melhor controle da rejeição, preservação mais adequada do fígado, profilaxia e tratamento de infecções secundárias e avanços em técnica cirúrgica, como doação inter vivos e transplante de segmentos hepáticos (split-liver), contribuíram para melhora da sobrevida e aumento do número de transplantes hepáticos na última década ${ }^{1-4}$. Durante o ano de 1999 , foram realizados cerca de 4.500 transplantes hepáticos nos Estados Unidos ${ }^{5}$ e 3.800 na Europa ${ }^{6}$. No Brasil, com a promulgação da nova lei de transplantes de órgãos e tecidos e a intensa discussão do assunto nos diversos segmentos da população, houve aumento de $171 \%$ no número de transplantes hepáticos nos últimos cinco anos ${ }^{7}$. $\mathrm{O}$ incremento foi de 133 transplantes em 1995 para 361 em $1999^{7}$.

Apesar da melhora dos resultados, as complicações vasculares permanecem como causa importante de morbidade, mortalidade e perda de enxerto pós-transplante ${ }^{8}$. A trombose da artéria hepática é a causa mais freqüente de retransplante hepático ${ }^{9-11}$. O nosso objetivo é apresentar as complicações vasculares arteriais e venosas observadas em um serviço de transplante hepático universitário brasileiro.

1. Professor Titular e Chefe dos Serviços de Cirurgia do Aparelho Digestivo e Transplante Hepático do HC-UFPR.

2. Médicos dos Serviços de Cirurgia do Aparelho Digestivo e Transplante Hepático do HC-UFPR.

3. Estagiário do Serviço de Transplante Hepático do HC-UFPR.

4. Acadêmica de Medicina da UFPR.

Recebido em 7/7/2000

Aceito para publicação em 12/9/2000

Trabalho realizado no Serviço de Transplante Hepático do Hospital de Clínicas da Universidade Federal do Paraná HC-UFPR. 


\section{MÉTODOS}

Foram revisados os prontuários de todos os pacientes submetidos a transplante hepático no Serviço de Transplante Hepático do Hospital de Clínicas da Universidade Federal do Paraná no período de setembro de 1991 a janeiro de 2000 .

Foram analisados dados referentes ao sexo, à idade, aos medicamentos utilizados e às doenças associadas dos receptores; cirurgias previamente realizadas pelos receptores; indicação do transplante hepático; número e tipo de complicação vascular; método utilizado para o diagnóstico da complicação; evolução clínica e tratamento dos pacientes; data e tempo de cirurgia; intercorrências durante o procedimento e outras complicações associadas. As anastomoses vasculares foram realizadas a olho nu ou com lupas cirúrgicas ( 3,5 vezes de aumento), exceto nas anastomoses arteriais dos transplantes inter vivos, em que foi utilizado microscópio cirúrgico (10-20x). Utilizou-se o teste do qui-quadrado para análise estatística, considerando nível de significância com $\mathrm{p}<0,05$.

\section{RESULTADOS}

Foram realizados 169 transplantes de fígado, sendo quatro inter vivos e nove retransplantes. As indicações dos transplantes estão listadas na Tabela 1. As indicações mais comuns foram hepatite pelo vírus $\mathrm{C}$ e cirrose criptogenética. Dos nove retransplantes, um foi devido à rejeição crônica e oito à trombose da artéria hepática, sendo que um desses pacientes apresentava trombose da veia porta associada.

Tabela 1

Indicações do transplante hepático $(\mathrm{N}=169)$

\begin{tabular}{l|c|c}
\hline Indicação & $N$ & $\%$ \\
\hline Cirrose por vírus da hepatite C & 35 & 20,7 \\
Cirrose criptogenética & 19 & 11,2 \\
Cirrose biliar primária & 15 & 8,9 \\
Cirrose alcoólica & 13 & 7,7 \\
Cirrose por hepatite auto-imune & 12 & 7,1 \\
Trombose a. hepática (retransplante) & 8 & 4,7 \\
Outras & 67 & 39,6
\end{tabular}

Um total de 24 complicações vasculares $(14,3 \%)$ foi identificado em 22 pacientes $(13,01 \%)$ (Tabela 2$)$. Um paciente apresentou duas complicações vasculares (trombose da artéria hepática e da veia porta) e um outro evoluiu para nova trombose da artéria hepática três semanas após ter sido submetido a retransplante por trombose da artéria hepática. A complicação vascular mais comum foi a trombose da artéria hepática, seguida da trombose da veia porta. Complicações da veia cava inferior infra ou supra-hepática foram incomuns, ocorrendo em um total de três casos $(1,8 \%)$.
Tabela 2

Complicações vasculares pós-transplante hepático

\begin{tabular}{lrc}
\hline Complicação & $N$ & $\%$ \\
\hline Trombose artéria hepática & 15 & 8,9 \\
Trombose veia porta & 4 & 2,4 \\
Estenose veia porta & 2 & 1,2 \\
Estenose veia cava inferior infra-hepática & 2 & 1,2 \\
Estenose veia cava inferior supra-hepática & 1 & 0,6 \\
\hline Total & 24 & 14,3 \\
\hline
\end{tabular}

A idade dos pacientes variou de sete meses a 64 anos, com uma média de 38,2 anos. Houve maior predomínio de complicações vasculares em crianças $(26,06 \%)$ em relação aos adultos $(13,14 \%)$, com p<0,05. Quando foi considerado somente o grupo pediátrico não houve diferença na incidência de complicações vasculares entre as maiores $(23 \%)$ e as menores $(28 \%)$ de $10 \mathrm{~kg}$. Não foi observada complicação vascular nos quatro transplantes inter vivos.

Quanto ao sexo ocorreram 14 complicações no sexo masculino $(14,9 \%)$ e dez no feminino $(15,1 \%)$, não havendo diferença estatística entre os dois grupos.

Quanto à- presença de doenças associadas que pudessem aumentar a incidência de complicações vasculares, três pacientes tinham diabetes mellitus e dois hipertensão arterial sistêmica.

Com relação à data da realização dos transplantes, notou-se que a percentagem de complicações vasculares durante os primeiros 40 casos (15\%) (curva de aprendizado da equipe cirúrgica) foi similar à dos demais 129 $(13,9 \%)$. Quanto ao tempo cirúrgico, não se observou diferença no número de complicações vasculares quando este foi maior $(16 \%)$ ou menor $(13,1 \%)$ do que sete horas.

Quanto à apresentação clínica, sete pacientes tinham complicações biliares e disfunção do enxerto; dois somente complicações biliares, dois complicações biliares e sepse; três disfunção mínima do enxerto; dois bacteremia e sepse; dois insuficiência hepática e sepse; um disfunção de múltiplos órgãos e sistemas e tr6es eram assintomáticos.

O método diagnóstico utilizado foi somente ecodoppler em dez pacientes, ecodoppler seguido de arteriografia (Figura 1) em sete pacientes e somente arteriografia em três pacientes. Os outros dois pacientes tiveram diagnóstico confirmado somente durante necropsia.

Dos pacientes com trombose da artéria hepática, um foi submetido à angioplastia, um foi reoperado para realização de trombectomia e oito para realização de retransplante. Esta conduta também foi indicada em outros cinco pacientes que foram a óbito enquanto aguardavam o procedimento. Dos pacientes com trombose da veia porta, dois foram a óbito, um foi submetido à colocação de prótese (stent) na via porta por via percutânea (Figura 2) e um a tratamento conservador. Os pacientes com estenose da veia porta e da veia cava inferior infra e supra-hepática foram submetidos a tratamento conservador com heparina e cumarínico, com boa evolução (Figura 3). 


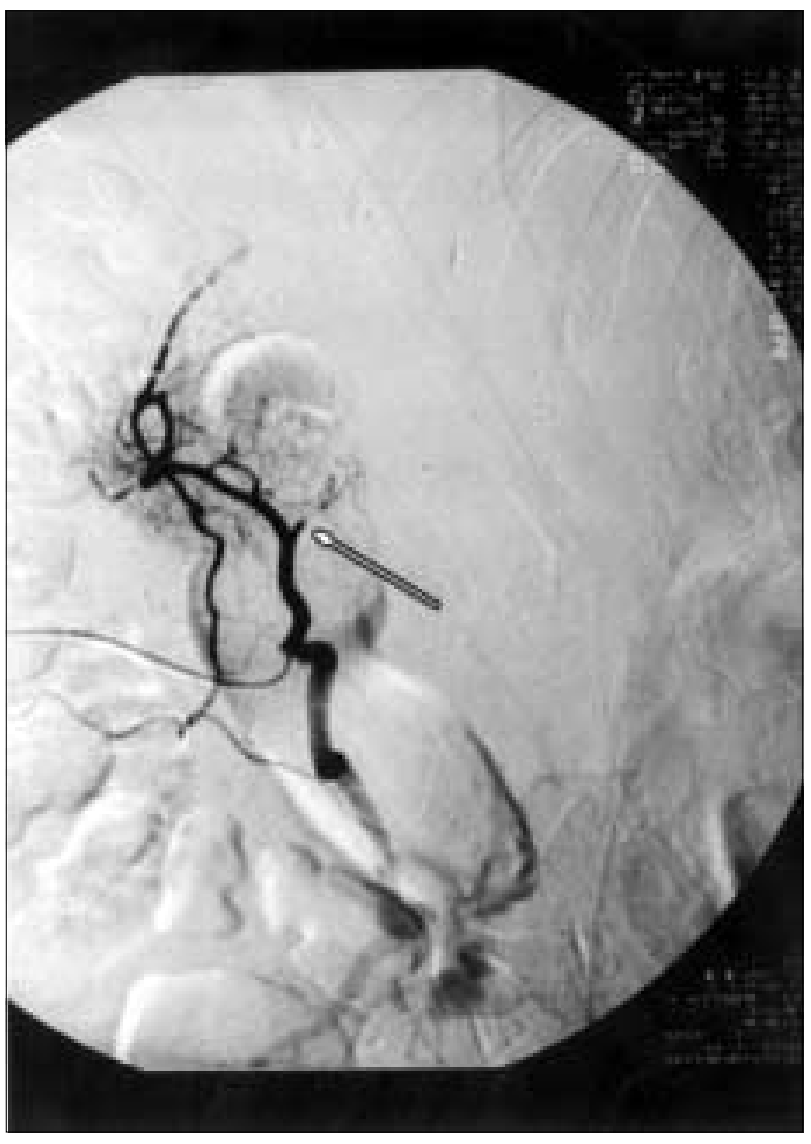

Figura 1 - Arteriografia do tronco celíaco evidenciando trombose da artéria hepática (seta).

\section{DISCUSSÃO}

Várias complicações vasculares podem ocorrer após o transplante hepático e são causa de elevadas morbidade, mortalidade e perda de enxerto ${ }^{12-14}$. A trombose da artéria hepática é a complicação vascular mais comum e é a segunda complicação grave mais comum, após as infecções ${ }^{15-1} \cdot{ }^{7}$ No grupo pediátrico é a complicação grave mais comum ${ }^{15-19}$. Devido ao pequeno diâmetro da artéria nas crianças, a trombose da artéria hepática é mais comum no grupo pediátrico (15-20\%) do que em adultos (5 a $10 \%)^{16,17}$. Na nossa experiência, as complicações vasculares foram duas vezes mais comuns nas crianças do que nos adultos. Entretanto, possivelmente pelo pequeno número de transplantes em crianças, não observamos diferença na incidência dessas complicações entre crianças menores e maiores do que $10 \mathrm{~kg}$. Como todos os vasos colaterais dos vários ligamentos hepáticos são seccionados durante a retirada do fígado para o transplante, a circulação arterial do fígado transplantado depende totalmente da artéria hepática ${ }^{15}$. Assim, trombose da artéria hepática causa isquemia e lesão irreversível do fígado ${ }^{15}$. As árvores biliares intra-hepática e extra-hepática do fígado transplantado são supridas somente por ramos da artéria hepática e, na presença de trombose desta, ocorre isquemia e necrose das vias biliares ${ }^{15}$.
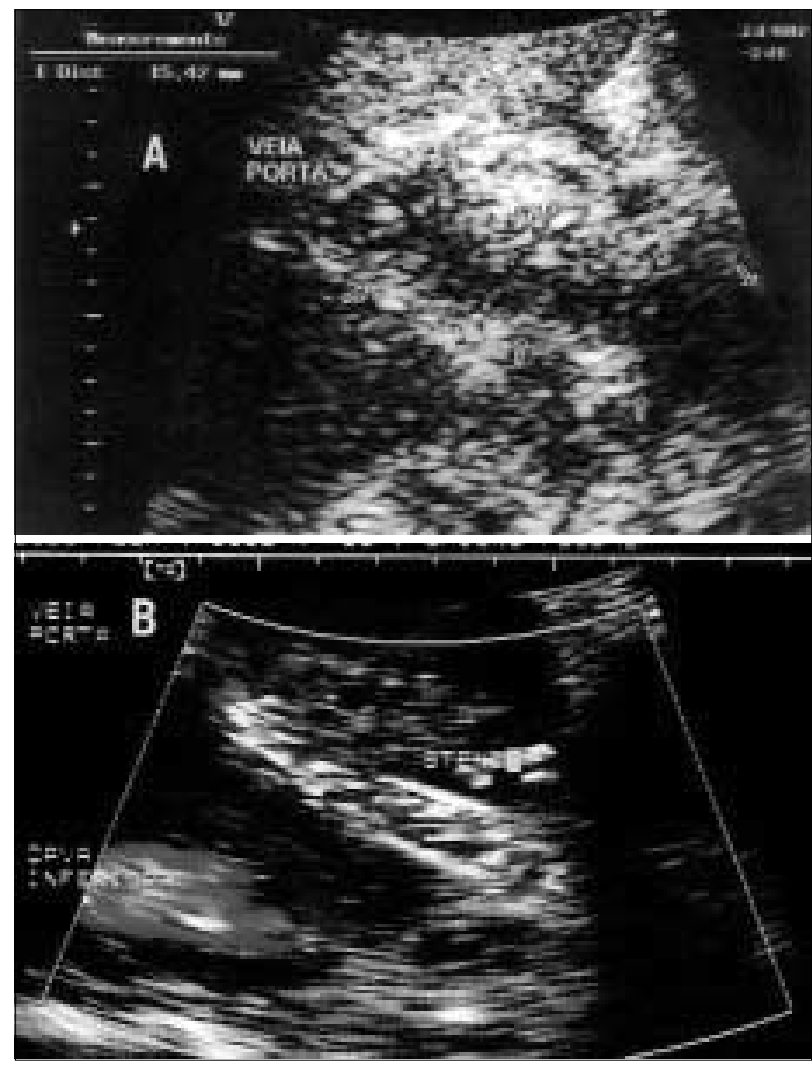

Figura 2 - Ecodoppler evidenciando trombose da veia porta antes $(A)$ e após $(B)$ a colocação de uma prótese endovascular.

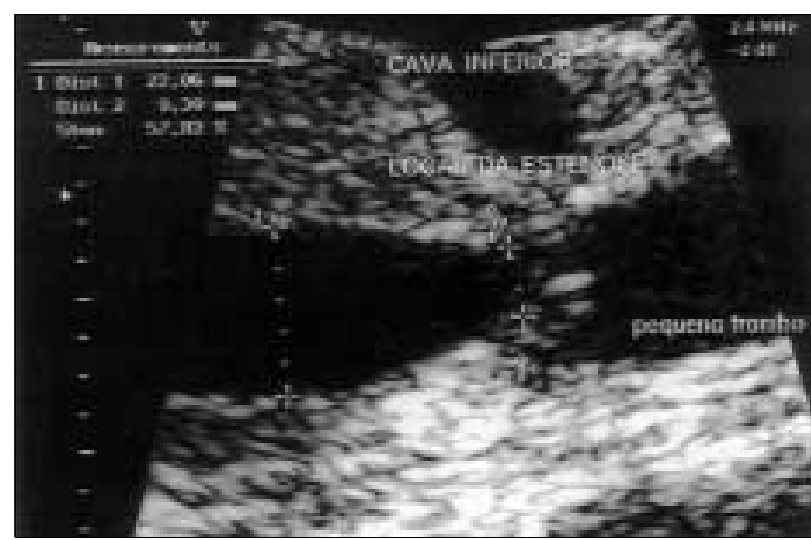

Figura 3 - Ecodoppler da veia cava inferior infra-hepática evidenciando estenose de 50\%, com um pequeno trombo.

As manifestações de trombose da artéria hepática variam desde ausência de sintomas até a ocorrência de choque séptico secundário à gangrena hepática fulminante $11,15,16$. Os pacientes podem apresentar um dos quatro grupos de sintomas: 1) Insuficiência hepática fulminante com aumento rápido e elevado das transaminases e bilirrubinas e coagulopatia. A necrose maciça do fígado é acompanhada de sepse, febre, encefalopatia hepática, coagulopatia, hipotensão e dispnéia; 2) Bacteremia recidivante com fe- 
bre, elevação discreta das enzimas hepáticas, leucocitose e hemoculturas positivas; 3 ) Complicações biliares, sendo as mais freqüentes colangite, estenose biliar, extravasamento de bile e bilioma (cavidade intra-hepática de dimensões variáveis de poucos a mais de $10 \mathrm{~cm}$ ); 4) Assintomáticos com nenhuma ou mínima alteração dos exames de função hepática.

O ecodoppler colorido é o primeiro exame a ser solicitado e tem uma precisão de 80 a $95 \%{ }^{14}$. A maioria dos serviços, como o nosso, realiza este exame de rotina nos primeiros dias de pós-transplante para avaliar a permeabilidade da artéria e diagnosticar sua trombose o mais precocemente possível, caso ocorra. A arteriografia é indicada para confirmar o diagnóstico.

O tratamento depende do tempo em que a trombose ocorreu após o transplante. Nos casos precoces, angioplastia percutânea ou revisão cirúrgica da anastomose arterial de emergência estão indicadas ${ }^{9}$. Entretanto, a maioria necessita retransplante. Muitos morrem, como cinco de nossos pacientes, enquanto aguardam o procedimento na fila de espera. Somente algumas crianças, que desenvolvem uma rede arterial colateral efetiva, não necessitam retransplante ${ }^{16}$

A incidência de trombose da veia porta é muito menor do que a da artéria hepática. Ocorre em 1 a $2 \%$ dos pacientes ${ }^{20}$. É mais freqüente em pacientes que tinham trombose da veia porta previamente ao transplante ${ }^{11,16}$. Defeitos técnicos, condições associadas à hipercoagulação sangüínea e esplenectomia (causa redução do fluxo sangüíneo e trombocitose) também predispõem a esta grave complicação ${ }^{11,16}$. A apresentação clínica depende se a trombose ocorre precoce ou tardiamente. Na presença de trombose precoce, as conseqüências são graves. O paciente geralmente apresenta disfunção hepática grave, instabilidade hemodinâmi- ca, insuficiência respiratória, ascite volumosa, varizes esofagianas e esplenomegalia ${ }^{8}$. Quando a trombose da veia porta é tardia, a função hepática é geralmente preservada e o paciente apresenta esplenomegalia e varizes esofagianas ${ }^{8}$.

O diagnóstico é confirmado por ecodoppler colorido. A fase venosa da arteriografia, a ressonância magnética e a venografia transportal podem ser necessárias ${ }^{12}$.

Se a trombose for diagnosticada precocemente, revisão da anastomose da veia porta está indicada. A colocação percutânea de uma prótese na veia porta pode ser efetiva, como observado em um de nossos casos. Nos pacientes em que o diagnóstico é estabelecido tardiamente e a função hepática está preservada, escleroterapia das varizes do esôfago ou, se necessário, a anastomose esplenorrenal podem ser suficientes para controlar a hemorragia das varizes do esôfago ${ }^{8}$. Nos demais casos, o retransplante é necessário ${ }^{16}$.

A trombose da veia cava é uma complicação incomum pós-transplante hepático, sendo relatada em cerca de $1 \%$ dos pacientes ${ }^{21}$. Entretanto, a mortalidade é elevada, cerca de $50 \%{ }^{21}$. As manifestações clínicas incluem ascite, hepatomegalia gigante, edema dos membros inferiores e insuficiência hepática e renal ${ }^{8}$. As causas mais comuns são defeito técnico e recidiva da síndrome de Budd-Chiari ${ }^{8}$. O ecodoppler colorido estabelece o diagnóstico. Venocavografia inferior pode ser necessária. Dilatação com balão, administração de uroquinase e uso de anticoagulantes por tempo prolongado podem ser eficazes ${ }^{21}$. Retransplante hepático é indicado nos casos de falha do tratamento anterior. Conclui-se deste estudo que as complicações vasculares são freqüentes após o transplante hepático, principalmente em crianças, e são associadas à elevada taxa de morbidade, mortalidade e retransplante.

\begin{abstract}
Background: The authors present the vascular complications observed in an academic Brazilian hepatic transplantation division. Methods: Hospital charts of all patients who were submitted to a liver transplantation between of September 1991 and January 2000, were evaluated to determine vascular complications and to correlated them to clinical data and surgical procedures. Results: Of a total of 169 tranplantations performed, four were from living related donors and 9 retransplantations. Twenty-four vascular complications (14,3\%) were identified in 22 patients $(13,0 \%)$, the most common being hepatic artery thrombosis (15 cases) and portal vein thrombosis (4 cases). Complications of the infra and suprahepatic vena cava were observed in three cases $(1,8 \%)$. Vascular complications were more common in children $(26,06 \%)$ than in adults $(13,14 \%)(p<0,05)$. From the patients with hepatic artery thrombosis, one was submitted to an angioplasty, one to a thrombectomy, while eight underwent retransplantation and five died waiting for retransplantation. Of the patients with portal vein thrombosis, two died, one submitted to an endovascular stent placement, and one to conservative treatment. Patients with stenosis of the portal vein and of the infra and suprahepatic inferior vena cava received a conservative treatment, with good clinical recovery. Conclusion: Vascular complications are frequent after liver transplantation, mainly with children. They are associated with an elevated rate of morbidity, mortality and retransplantation.
\end{abstract}

Key Words: Hepatic transplantation; Vascular complications; Venous thrombosis; Arterial thrombosis. 


\section{REFERÊNCIAS}

1. Raia S, Nery JR, Mies S - Liver transplantation from live donors. Lancet 1989; 2:497.

2. Carone E, Chapchap P, Pugliese V et al. Transplante hepático com doador vivo familiar: técnica operatória no doador. Rev Col Bras Cir 1997; 24:235-241.

3. Perrilo R, Rakela J, Dienstag J, et al - Multicenter study of lamivudine therapy for hepatitis B after liver transplantation. Lamivudine Transplantation Group. JAMA 1999; 281:1381-1386.

4. Neuberger $\mathbf{J}$ - Transplantation for alcoholic liver disease: a perspective from Europe. Liver Transpl Surg 1998: 4:S51-S57.

5. Graham WK - Organ transplantation in the United States. Organs Tissues 1999; 2:83-90.

6. Matesanz R, Mühlbacher F, van Hallewizck B - International figures on organ donation \& transplantation. Organs Tissues 1999; 1:5-56.

7. Registro Brasileiro de Transplantes (ABTO) 1999, 5:5-58.

8. Mazariegos GV, Molmenti EP, Kramer DJ - Early complications after orthotopic liver transplantation. Surg Clin N Am 1999; 79:17-33.

9. Raby N, Karani J, Thomas S, et al - Stenoses of vascular anastomoses after hepatic transplantation: treatment with balloon angioplasty. AJR 1991; 157:167-171.

10. Shaw BW Jr, Gordon RD - Hepatic retransplantation. Transplant Proc 1995; 17:264-271.

11. Karatzas T, Lykaki-Karatzas E, Webb M, et al - Vascular complications, treatment, and outcome following orthotopic liver transplantation. Transpl Proc 1997; 29:2853-2855.

12. Stafford-Johnson DB, Hamilton BH, Dong Q, et al - Vascular complications of liver transplantation: evaluation with Gadolinium-enhanced MR angiography. Radiology 1998; 207:153-160.

13. Millis JM, Seaman DS, Piper J, et al - Portal vein thrombosis and stenosis in pediatric liver transplantation. Transplantation 1996; 62:748-754.

14. Propeck PA, Scanlan KA - Reversed or absent hepatic arterial diastolic flow in liver transplants shown by duplex sonography: a poor predictor of subsequent hepatic artery thrombosis. AJR 1992; 159:1199-1201.
15. Coelho JCU, Wiederkehr JC, Campos ACL, et al - Cavidade biliar (bilioma) intra-hepática secundária à trombose tardia da artéria hepática após transplante hepático. Rev Col Bras Cir 1994; 21:29-31.

16. Marujo WC, Langnas AN, Wood RP, et al - Vascular complications following orthotopic liver transplantation: outcome and the role of urgent revascularization. Transpl Proc 1991; 23:1484-1486.

17. Orons PD, Hari AK, Zajko AB - Hepatic artery stenosis in liver transplant recipients: prevalence and cholangiographic appearance of associated biliary complications. AJR 1994; 165:1145-1149.

18. Coelho JUC, Wiederkehr JC, Campos ACL, et al - Pancreátite aiguë par le vírus varicella-zoster après une transplantation du foie. J Chir (Paris) 1994; 131:96-98.

19. Coelho JCU, Wiederkehr JC, Cat R, et al - Extrapyramidal disorder secondary to cytomegalovirus infection and toxoplasmosis after liver transplantation. Eur J Pediatr Surg 1996; 6:110-111.

20. Funaki B, Rosenblum JD, Leef JA, et al - Portal vein stenosis in children with segmental liver transplants: treatment with percutaneous transhepatic venoplasty. AJR 1995; 165:161-165.

21. Orons PD, Hari AK, Zajko AB, et al - Thrombolysis and endovascular stent placement for inferior vena caval thrombosis in a liver transplant recipient. Transplantation 1997; 64:1357-1361.

Endereço para correspondência:

Dr. Júlio Cezar V. Coelho

Rua Bento Viana, 1.140/2202

80240-110 - Curitiba-PR

Fone/Fax: 41-322-3789 